\title{
Palatal Rugae Patterns as an Adjuvant to Gender Determination in Forensic Identification Procedures in a Paediatric Population
}

\section{Jaisingh $\mathrm{R}^{1^{*}}$ Deshmukh $\mathrm{S}^{2}$ and Srilatha $\mathrm{KT}^{3}$}

${ }^{1}$ Final Year Post Graduate Student in MDS, Department of Pediatric Dentistry, JSS Dental College and Hospital, Mysuru, Karnataka, India

${ }^{2}$ Reader, Department of Pediatric Dentistry, JSS Dental College and Hospital, Mysuru, Karnataka, India ${ }^{3}$ Head of Department, Department of Pediatric Dentistry, JSS Dental College and Hospital, Mysuru, Karnataka, India

${ }^{*}$ Corresponding author: Jaisingh R, Final Year Post Graduate Student in MDS, Department of Pediatric Dentistry, JSS Dental College and Hospital, Mysuru, Karnataka, India, E-mail: rjaisingh@gmail.com

Citation: Jaisingh R (2016) Palatal Rugae Patterns as an Adjuvant to Gender Determination in Forensic Identification Procedures in a Paediatric Population. J Forensic Sci Criminol 4(3): 305. doi: 10.15744/23489804.4.305

Received Date: June 08, 2016 Accepted Date: June 29, 2016 Published Date: June 30, 2016

\begin{abstract}
Identification of an individual who is either living or dead is based on the assumption that all individuals are unique. Whenever an unidentified corpse is found, the individual is classified into characteristic groups and finally the various possibilities are narrowed down. In the present day scenario, countless number of heinous and inconceivable crimes and catastrophes have led to the need for new possibilities in identification of human remains. Most often whenever forensic identification is incomplete owing to the lack of availability of DNA, fingerprints or dental records, palatal rugae patterns can serve as a reliable adjunct to forensic identification procedures. Palatal Rugae can be compared to ones finger prints in the sense that they remain stable throughout life. The rugae patterns are unaffected by physical and chemical injuries and hence can serve as reliable tool in gender determination and forensic identification procedures. The aim of this study was to record the palatal rugae patterns in terms of shape length and orientation to assess gender variations in a paediatric population of six to twelve years. They were divided into two groups of equal males and females of age groups 6-9 years and 10-12 years respectively. The Thomas and Kotze classification of rugae was used for the analysis which was based on the linear dimension, shape and unification of the rugae. Study models 80 maxillary impressions were prepared using dental stone: 20 males (6-9 years), 20 females (6-9 years), 20 males (10-12 years), 20 females (10-12 years). The results revealed that primary rugae was the most commonly found pattern. Primary rugae are seen more in males of 10-12 years and this was found to be statistically significant. However, there were no statistically significant differences among the other rugae patterns among males and females in the two age groups. Wavy rugae are the most common rugae based on shape. Based on unification, divergent rugae were more common than convergent rugae.
\end{abstract}

Keywords: Palatal rugae; Gender determination, Forensic identification

\section{Introduction}

Determining an individual's identity is a complex process. Victim identification in a catastrophe or crime scene deems to be the primary objective for personal, legal and social reasons. The most commonly used sources for forensic identification are the human teeth, DNA matching and finger prints when visual identification of the individual is unclear. However when situations when one or more of the above sources are unavailable it is necessary to use an adjunct such as Rugoscopy or Palatal Rugae Analysis. According to morlang, fingerprints serve as the standard for human identification but this method will not be an option if there are no antemortem records or if the post mortem fingerprints are unavailable owing to fire, massive trauma or decomposition [1].

Palatine rugae are also called plica palatine transversae or rugae palatine. They are asymmetrical and irregular elevations found in the anterior one third of the palate. They develop around the third month of intrauterine life from the connective tissue of the palatine process of the maxillary bone. The growth and development of the rugae are controlled by mutual epithelio-mesenchymal interactions and specific extra cellular matrix molecules are spatially expressed. It develops from the lateral membrane of the incisal papilla in a transverse direction along the mid saggital plane. Palatal rugae can be used in medico legal identification processes as they are stable over time and iscomparable to an inviduals fingerprint [2]. Palatal rugae resist decomposition, remain stable with respect to age and they stay unaltered after trauma or surgical procedures as they are protected by the lip, cheeks, teeth and buccal pad of fat in case of burns and trauma [3]. Trauma, extreme finger sucking habit and pressure exerted during orthodontic treatment and dentures might contribute to changes in palatal rugae patterns. In a study by Hauser, et al. it has been reported that the rugae pattern does not change with time or age. The linear extent of anterior rugae does not increase after 10 years of age according to Van Der Linden. A quantitative characteristic such as shape, direction and unification remains unchanged all through 
life. Even between twins, it has been reported that the patterns are similar but not identical [4].

Palatal rugae are resistant to chemical injuries and can be said to be unique for every individual as it is consistent throughout life [5].

The present study is aimed at analyzing the palatal rugae variations in children from Karnataka, India with a primary objective to assess the variations based on gender and age between the groups.

\section{Materials and Methods}

The sample included 80 individuals from a paediatric population of 6-12 years and comprising of two groups of equal number males and females. First group containing equal number of males and females of 6-9 years (20 M, $20 \mathrm{~F})$ and the second group containing equal number of males and females of 10-12 years (20M, 20F). After obtaining informed consent, children within the specific age group visiting the dental Out Patient Department at Jagadguru Sri Sivarathreeshwara Dental College and Hospital (JSSDCH) were chosen. Children undergoing orthodontic appliance therapy and those having palatal defects were not included in the study.

The study was carried out for two months. Rugae patterns were recorded based on Thomas and Kotze classification which included the length, shape and unification patterns of rugae. A rugae length greater than $5 \mathrm{~mm}$ was referred to as primary rugae, those which have a length of 3-5 mm were called secondary rugae and fragmentary rugae are those having a length of 2-3mm. Based on the shape, Rugae can be classified as curved, wavy, straight and circular. Straight forms run directly from their origin to the termination, Curved forms are crescent shaped with a gentle curve. Wavy rugae are serpentine in appearance. Circular rugae were called so only if it were a continuous ring. Unification is said to be present if two rugae join either at their origin or termination. When two rugae have the same origin and diverge out, they are referred to as diverging rugae. When two rugae having different origins medially later have a common termination laterally are referred to as converging rugae [4]. A single investigator carried out the oral examination of the subjects. The investigator underwent training for two successive days. After obtaining an ethical clearance certificate from the institutional ethical committee, a total of 80 impressions were recorded using alginate impression material and dental stone casts were prepared. Each cast was analysed twice to minimize intra - observer variations. The rugae patterns were demarcated on the Dental stone casts with the help of a marker and the linear extent of the rugae were measured using a hairspring divider. A magnifying glass was used to assess the rugae shape and unification. The data thus obtained was prepared for statistical analysis using SPSS version 21 (Figure 1 and 2).

Statistical analysis was done using an independent $\mathrm{t}$ - test.

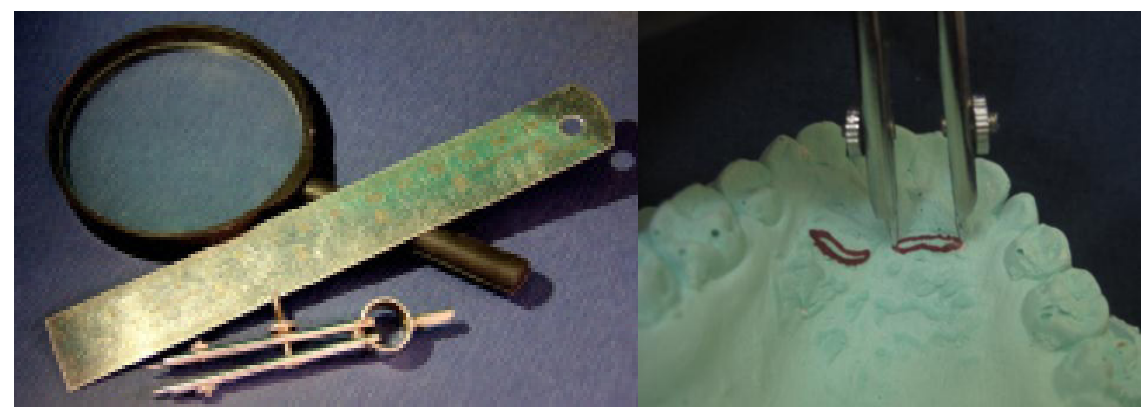

Figure 1: A) showing the armamentarium used in the measurement of palatal rugae. B) The process of measuring the linear extent of the rugae

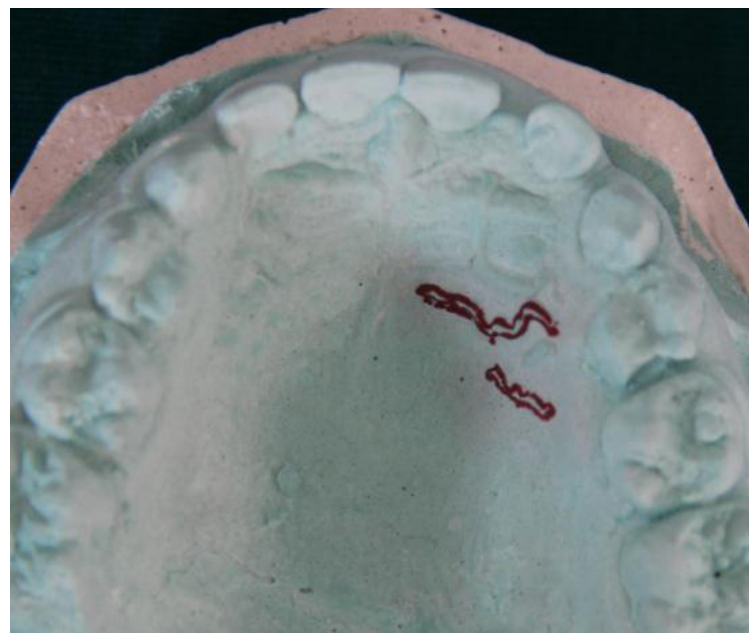

A) Primary rugae

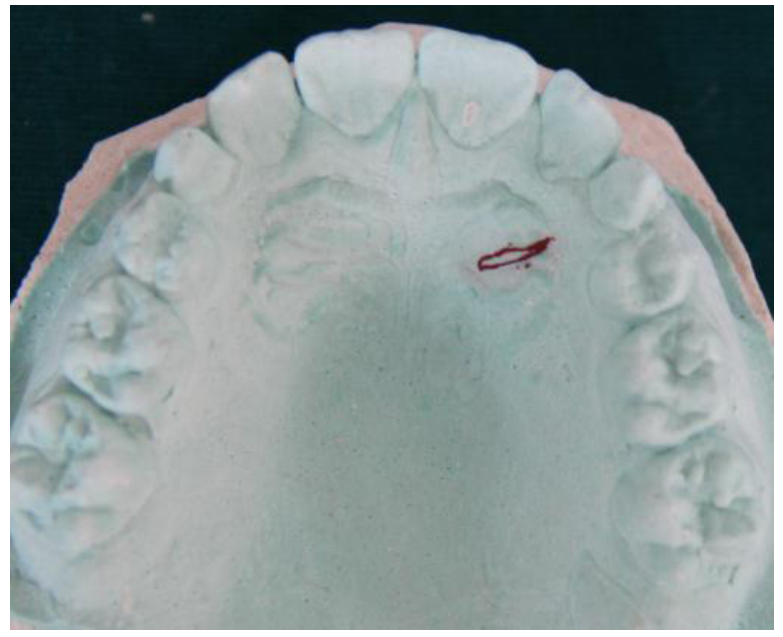

B) Secondary Rugae 


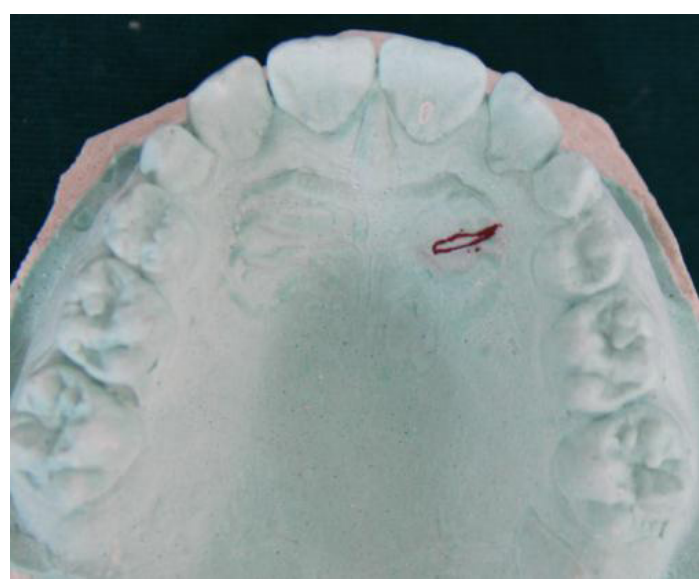

C) Straight rugae

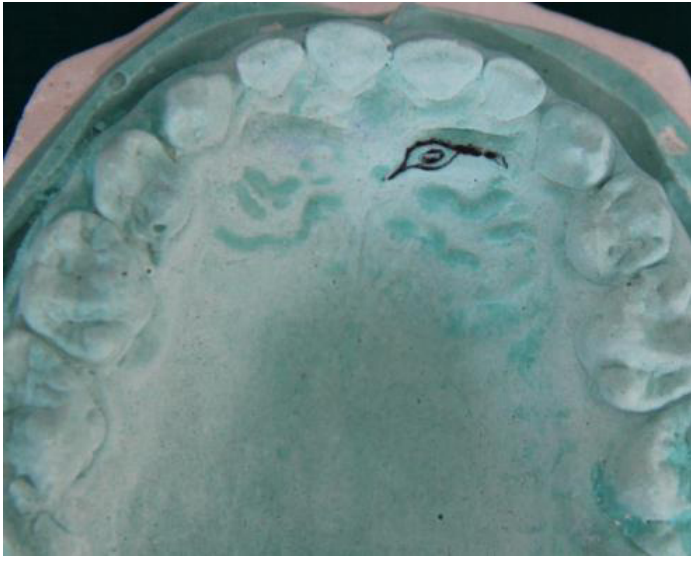

E) Circular form

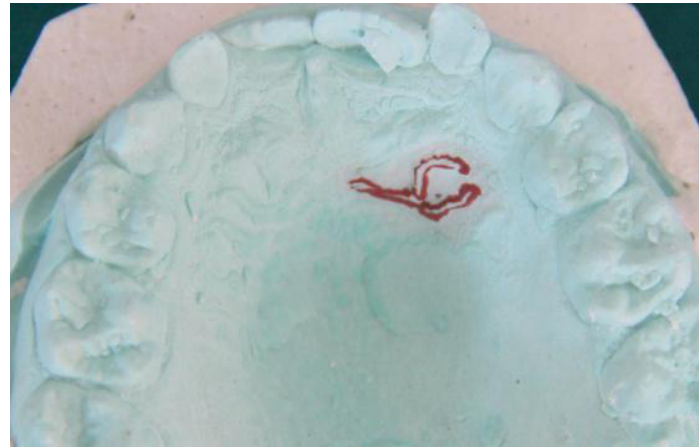

G) Converging form

Figure 2: Different types of rugae based on Thomas and Kotze classification

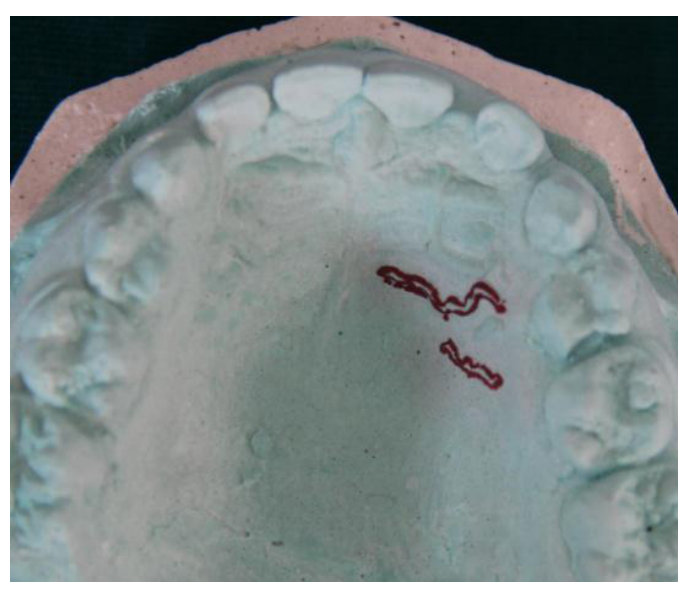

D) Wavy form

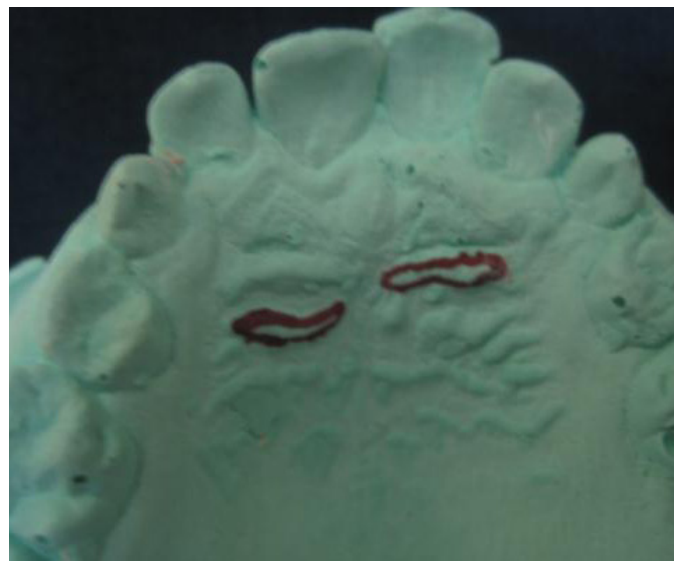

F) Curved form

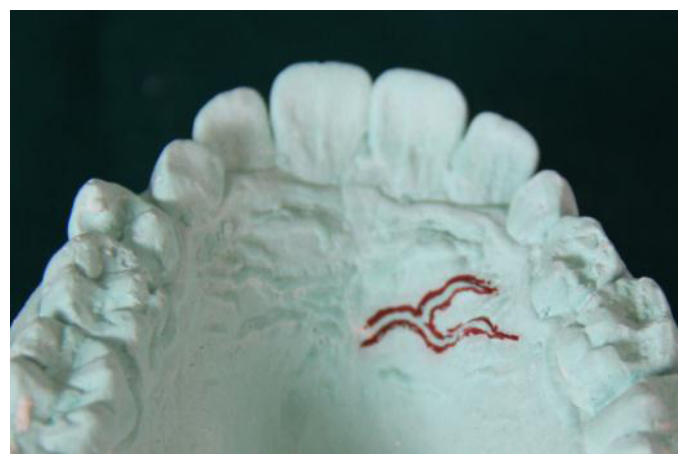

H) Diverging form

\section{Results}

The study was done to assess gender variations in rugae patterns a paediatric population of equal males and females of 6-9 years and $10-12$ years.

\section{Comparison of the total number of rugae in males and females}

A total of 1207 palatal rugae patterns of a total of 80 children were recorded. The total number of rugae of 40 males and 40 females of the two groups were found to be higher in males (647) compared to females (560). No significant differences were observed between the total number of rugae of males and females. The total number of primary rugae was found to be higher in males in the 6-9 and 10-12 groups. However, the primary rugae were significantly higher in males of 10-12 years (Table 1) [6,7].

\begin{tabular}{|c|c|c|c|c|c|}
\hline GENDER & $\mathbf{n}$ & TOTAL RUGAE & MEAN & SD & SE \\
\hline Males & 40 & 339 & 8.55 & 1.66 & 0.37 \\
\hline Females & 40 & 309 & 7.55 & 1.50 & 0.35 \\
\hline
\end{tabular}




\section{Comparison Based on the rugae shape}

Out of the 1207 palatal rugae recorded, 447(37\%) rugae were wavy and 277(23\%) were curved. Wavy form was the most predominate shape in the two groups. The wavy form was seen more in males-234(52.3\%) than females (47.6\%). The curved pattern of rugae was also more in males-146(52.7\%) than in females-131(47.2\%). Straight rugae were only 90(7.4\%) of the total. Circular shape rugae were only $0.74 \%$ of the total rugae found. Based on unification, divergent rugae $(2.4 \%)$ were more than converging rugae (1.24\%). Divergent rugae were more common in females than males.

These findings are in accordance with the studies done by Kapali, et al., Nayak, et al. and Sharma which states that the most commonly found rugae patterns are the wavy and curved forms, whereas circular and straight forms were infrequently found. One study showed that the converging type of rugae unification was found more in females than males contrary to the results obtained in this study [8-11].

\section{Conclusion}

The following results were obtained from the present study. Primary rugae is the most prevalent form of rugae in terms of length followed by secondary rugae as shown in Figure 3. The mean number of primary rugae was significantly higher in males of 1012 years. Wavy form is the most predominantly found rugae based on shape followed by the curved form. Based on unification, divergent forms are more common than convergent forms (Figure 4 and 5).

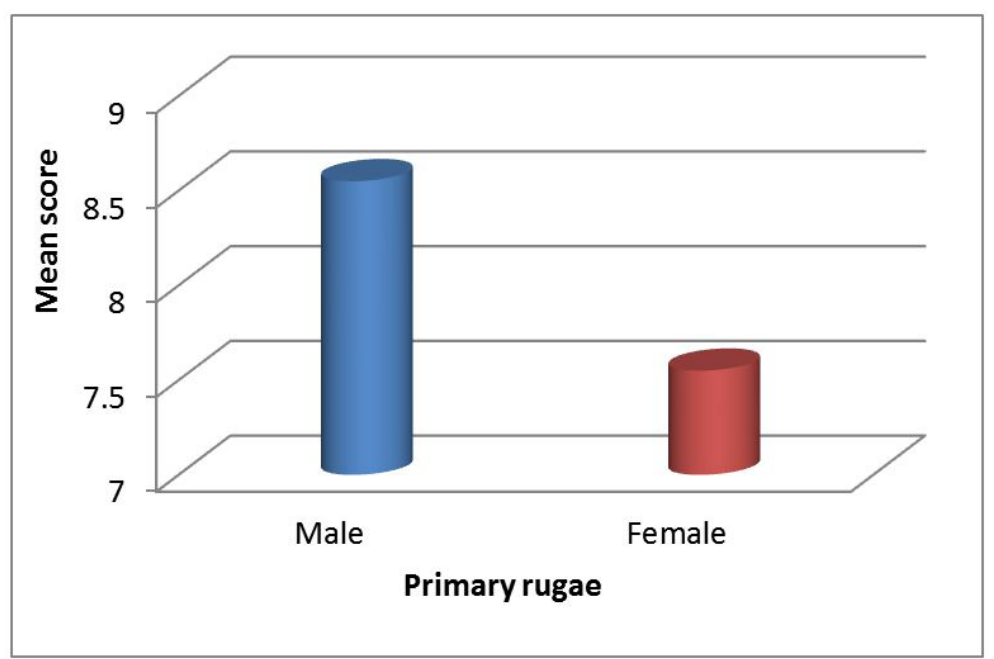

$\mathrm{P}=0.054$

Figure 3: Primary rugae is the most commonly seen type of rugae and

is significantly higher in males compared to females

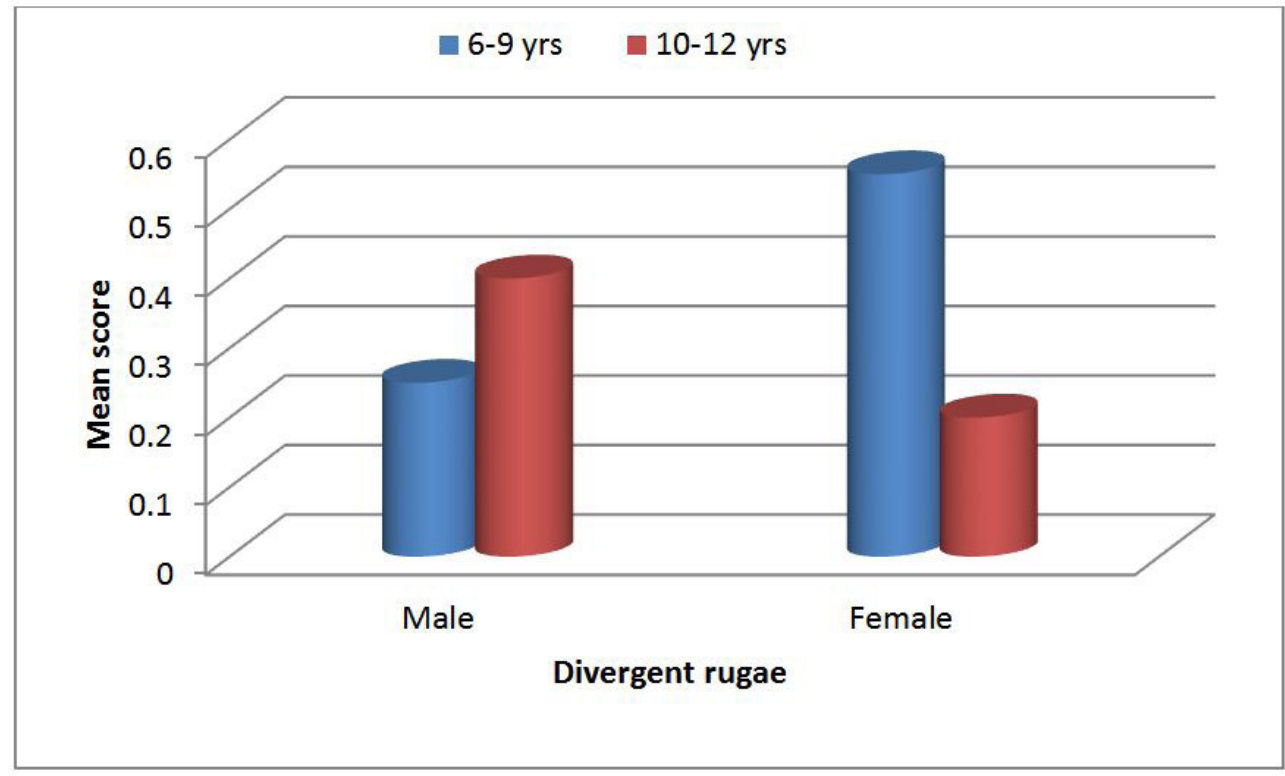

Figure 4: Based on the unification, divergent rugae was more common than the convergent type and was higher in females than males 
MALES
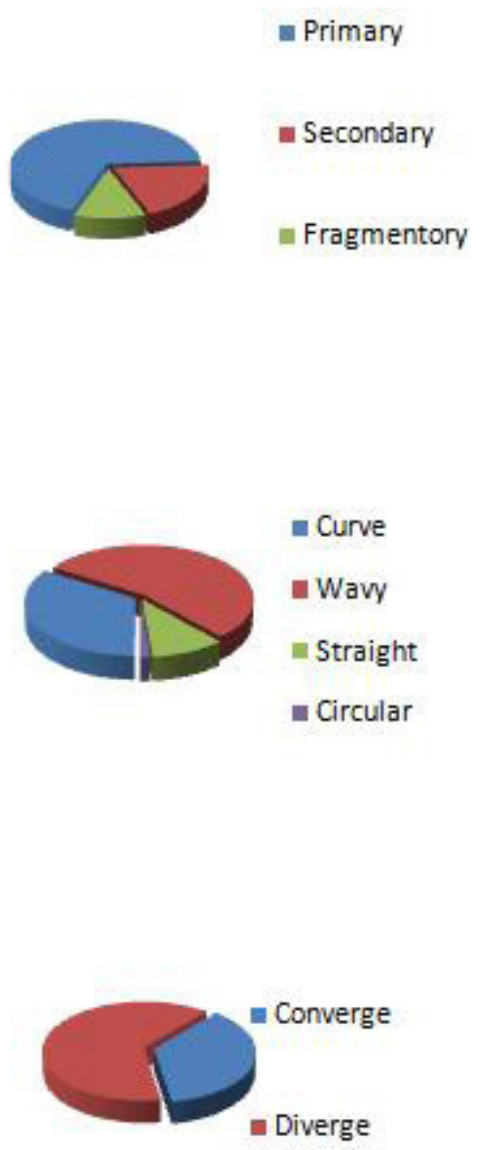

FEMALES
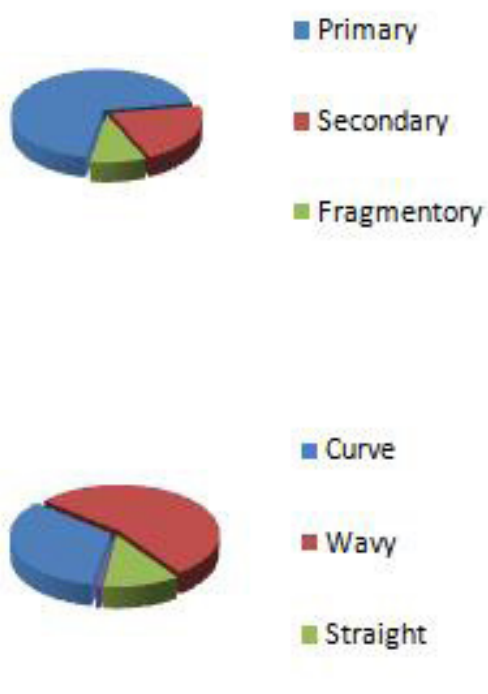

- Circular

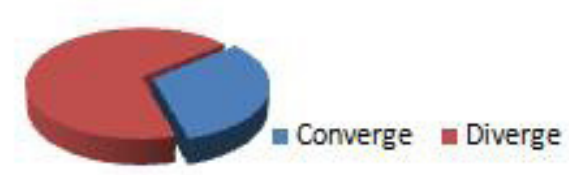

Figure 5: Illustration of rugae patterns in males and females

\section{Discussion}

The study of the palatal rugae patterns are in other words called as palatal rugoscopy or palatoscopy. Human identification still comes across as a major challenge faced by man and the possibility of a new, cost effective and reliable adjunct would definitely be of great help.

The patterns of the rugae are due to the presence of core fibres running antero-posteriorly in concentric circles beneath each rugae. The connective tissues of the rugae are composed of fibroblasts and collagen which determines its specific pattern. The structural units of these rugae are glycosaminoglycans which are hydrophilic in nature and can be attributed to the swollen and stable appearance of the tissue [6].

Previous study mainly by Almeida, et al. and Bailey, et al. comparing the rugae patterns pre and post extractions and orthodontic treatment did not show any significant variations thereby confirming the stability of the rugae over time [7,12,13]. One study claimed that changes do occur during orthodontic interventions however, the matching of the rugae patterns before and after treatment did not show any significant changes [14].

A study comparing the rugae patterns of two populations of India categorized as southern and western groups respectively revealed that the curvy and wavy forms were the most prevalent and was statistically significant. However gender variations were not seen $[8]$.

A study comparing the rugae patterns of the coorg and malayalee populations of south India revealed that the wavy form was the most prevalent among the coorg population. There was a statistically significant difference between the wavy form and unifications in the two population groups. A Gender variation in the primary rugae was seen in the malayali population group with primary rugae being significantly higher in males than females [15]. 
Regarding the rugae shape, the findings of the present study are in accordance with the studies done by Kapali, et al. Nayak, et al. and Sharma which states that the most commonly found rugae patterns are the wavy and curved forms, whereas circular and straight forms were infrequently found. One study showed that the converging type of rugae unification was found more in females than males contrary to the results obtained in this study [8-11].

In accordance with the results of our study, a study done by Chopra, et al. in 2013 showed the presence of increased number of divergent rugae in females in comparison to males [16,17]. Some of the studies done on adult patients found the total and mean number of rugae to be higher in males compared to female,. However in our study, total number of rugae was found to be higher in males than in females falling in the 6-12 year age group. Studies done in the past by Saraf, et al. found the total and mean number of rugae to be higher in males than females Similar to the findings in the present study $[4,18]$.

\section{References}

1. Morlang WM (1982) Forensic dentistry. Aviat Space Environ Med : 27-34

2. Hermosilla Venegas V, San Pedro Valenzuela J, Cantin Lopez M, Suazo Galdames IC (2009) palatal rugae: systematic analysis of its shape and dimensions for use in human identification. Int J Morphol 27: 819-25.

3. Limson KS, Julian R (2004) Computerized recording of the palatal rugae pattern and an evaluation of its application in forensic identification. J Forensic Odontostomatol 22: 1-4.

4. Saraf A, Bedia S, Indurkar A, Degwekar S, Bhowate R (2011) Rugae patterns as an adjunct to sex differentiation in forensic identification. J Forensic Odontostomatol 29: 14-9.

5. Patil MS, Patil SB, Acharya AB (2008) Palatine rugae and their significance in clinical dentistry: a review of the literature. J Am Dent Assoc 139: 1471-8.

6. Indira AP, Gupta M, David MP (2012) Palatal rugae patterns for establishing individuality. J Forensic Dent Sci 4: 2-5.

7. Shetty D, Juneja A, Jain A, Khanna KS, Pruthi N, et al. (2013) Assessment of palatal rugae pattern and their reproducibility for application in forensic analysis. J Forensic Dent Sci 5: 106-9.

8. Kapali S, Townsend G, Richards L, Parish T (1997) Palatal rugae patterns in Australian Aborigines and Caucasians Aust Dent J $42: 129-33$.

9. Nayak P, Acharya AB, Padmini AT, Kaveri H (2007) Differences in the palatal rugae shape in two populations of India. Arch Oral Biol 52: 977-82.

10. Sharma P, Saxena S, Rathod V (2009) Comparative reliability of cheiloscopy and palatoscopy in human identification Indian J Dent Res $20: 453-7$.

11. Faisal MF, Saleh MA, Yousef F (2001) Talic Rugae pattern in a Saudi population sample of males and females Saudi Dental J 13: 1-4.

12. Almeida MA, Phillips C, Kula K, Tulloch C (1995) Stability of the palatal rugae as landmarks for analysis of dental casts. Angle Orthod 65:43-8.

13. Bailey LT, Esmailnejad A, Almeida MA (1996) Stability of the palatal rugae as landmarks for analysis of dental casts in extraction and nonextraction cases. Angle Orthod 66: 73-8.

14. Bansode SC, Kulkarni MM (2009) Importance of palatal rugae in individual identification. J Forensic Dent Sci 1: 77-81.

15. Shetty DK, Machale PS, Savant SC, Taqi SA (2013) Comparison of palatal rugae patterns in Kodava and Malayalee populations of South India. J Forensic Dent Sci 5: 85-9.

16. O'Shaughnessy PE (2001) Introduction to forensic science. Dent Clin North Am 45: 217-27.

17. Chopra A, Rao NC, Gupta N, Vashisth S (2013) Palatal rugae and arch length: A tool in gender determination. Univ Res J Dent 3: 54-9.

18. Kamala R, Gupta N, Bansal A, Sinha A (2011) Palatal Rugae Pattern as an aid for personal identification: A Forensic Study. J Indian Acad oral Med Radiol 23: $173-8$.

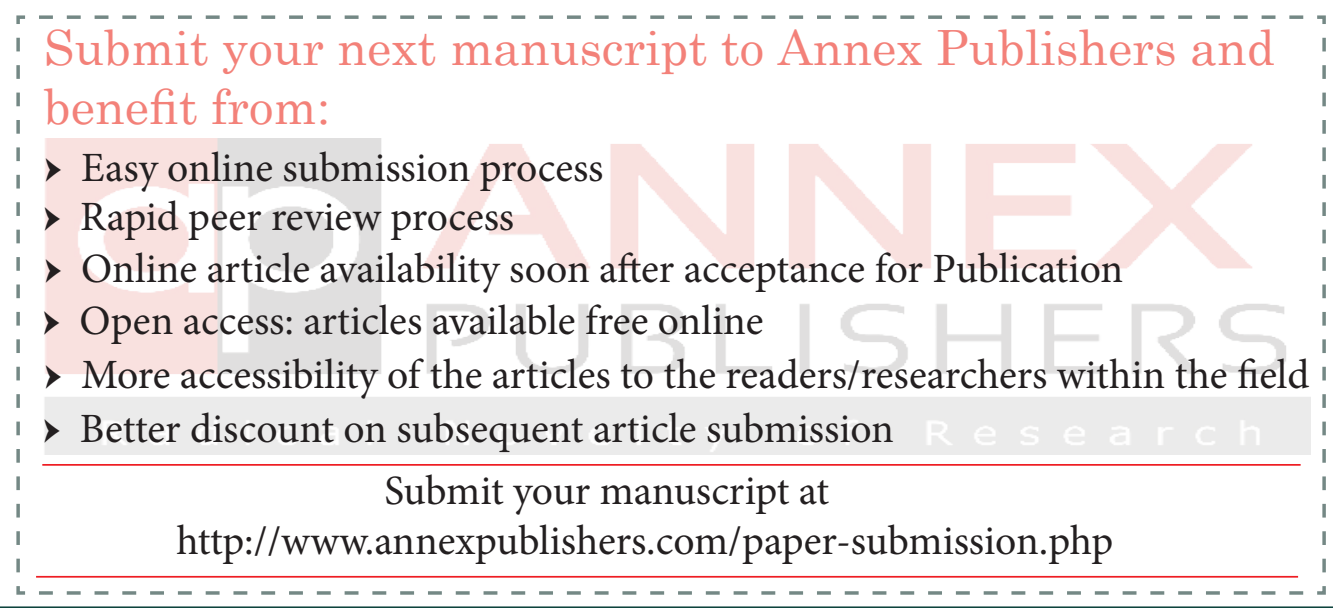

